\title{
TRACE AND EXTENSION THEOREMS RELATING BESOV SPACES TO WEIGHTED AVERAGED SOBOLEV SPACES
}

\author{
ARIEL BARTON
}

Abstract. There are known trace and extension theorems relating functions in a weighted Sobolev space in a domain $\Omega$ to functions in a Besov space on the boundary $\partial \Omega$. We extend these theorems to the case where the Sobolev exponent $p$ is less than one by modifying our Sobolev spaces to consider averages of functions in Whitney balls. Averaged Sobolev spaces are also of interest in the applications in the case where $p>1$, and so we also provide trace and extension results in that case. Finally, we provide some comparable results for Neumann traces and extensions.

Mathematics subject classification (2010): 46E35. ary values.

Keywords and phrases: Besov spaces, weighted Sobolev spaces, traces, extensions, Neumann bound-

\section{REFERENCES}

[1] Vilhelm Adolfsson And Jill PiPher, The inhomogeneous Dirichlet problem for $\Delta^{2}$ in Lipschitz. domains, J. Funct. Anal. 159, 1 (1998), 137-190.

[2] Mikhail AgRanovich, On the theory of Dirichlet and Neumann problems for linear strongly elliptic systems with Lipschitz domains, Funktsional. Anal. i Prilozhen. 41, 4 (2007), 1-21, 96. English translation: Funct. Anal. Appl. 41, 4 (2007), 247-263.

[3] Tosio Aoki, Locally bounded linear topological spaces, Proc. Imp. Acad. Tokyo, 18 (01942), 588 594.

[4] P. AusChER AND M. QAFSAOUI, Equivalence between regularity theorems and heat kernel estimates for higher order elliptic operators and systems under divergence form, J. Funct. Anal. 177, 2 (2000), 310-364.

[5] Pascal Auscher and Mihalis Mourgoglou, Boundary layers, Rellich estimates and extrapolation of solvability for elliptic systems, Proc. Lond. Math. Soc. (3), 109, 2 (2014), 446-482.

[6] ARIEl BARTON, Perturbation of well-posedness for higher-order elliptic systems with rough coefficients, http://arxiv.org/abs/1604.00062.

[7] ARIEL BARTON, Extrapolation of well-posedness for higher-order elliptic systems with rough coeffcients, http://arxiv.org/abs/1708.05079.

[8] ARIEL BARTON, Gradient estimates and the fundamental solution for higher-order elliptic systems with rough coefficients, Manuscripta Math. 151, 3-4 (2016), 375-418.

[9] Ariel Barton, Steve Hofmann, and Svitlana Mayboroda, Square function estimates on layer potentials for higher-order elliptic equations, Math. Nachr. 290, 16 (2017), 2459-2511.

[10] Ariel Barton, Steve Hofmann, and Svitlana Mayboroda, The Neumann problem for higher order elliptic equations with symmetric coefficients, to appear, Math. Ann.

[11] Ariel Barton ANd SVitlana Mayboroda, Higher-order elliptic equations in non-smooth domains: a partial survey, In Harmonic analysis, partial differential equations, complex analysis, Banach spaces, and operator theory, vol. 1, vol. 4 of Assoc. Women Math. Ser., pages 55-121. Springer, [Cham], 2016.

[12] ARIEL BARTON AND SVITLANA MAYBORODA, Layer potentials and boundary-value problems for second order elliptic operators with data in Besov spaces, Mem. Amer. Math. Soc. 243 (1149) (2016), $\mathrm{v}+110$. 
[13] Kevin Brewster And Marius Mitrea, Boundary value problems in weighted Sobolev spaces on Lipschitz manifolds, Mem. Differ. Equ. Math. Phys. 60 (2013), 15-55.

[14] S. Campanato, Sistemi ellittici in forma divergenza. Regolarità all'interno. Quaderni, [publications], Scuola Normale Superiore Pisa, Pisa, 1980.

[15] Jonathan Cohen And John Gosselin, Adjoint boundary value problems for the biharmonic equation on $C^{1}$ domains in the plane, Ark. Mat. 23, 2 (1985), 217-240.

[16] BJÖRn DAhlberg, Poisson semigroups and singular integrals, Proc. Amer. Math. Soc. 97, 1 (1986), 41-48.

[17] Ingrid Daubechies, Orthonormal bases of compactly supported wavelets, Comm. Pure Appl. Math. 417 (1988), 909-996.

[18] Eugene Fabes, Osvaldo Mendez, and Marius Mitrea, Boundary layers on Sobolev-Besov spaces and Poisson's equation for the Laplacian in Lipschitz domains, J. Funct. Anal. 1592 (1998), 323-368.

[19] Michael Frazier And Björn Jawerth, Decomposition of Besov spaces, Indiana Univ. Math. J. 344 (1985), 777-799.

[20] Steve Hofmann, Svitlana Mayboroda, and Mihalis Mourgoglou, Layer potentials and boundary value problems for elliptic equations with complex $L^{\infty}$ coefficients satisfying the small Carleson measure norm condition, Adv. Math. 270 (2015), 480-564.

[21] Steve Hofmann, Marius Mitrea, And Andrew Morris, The method of layer potentials in $L^{p}$ and endpoint spaces for elliptic operators with $L^{\infty}$ coefficients, Proc. Lond. Math. Soc. (3), 1113 (2015), 681-716.

[22] BJÖRn JAWERTH, The trace of Sobolev and Besov spaces if $0<p<1$, Studia Math. 62, 1 (1978), 65-71.

[23] David Jerison And Carlos Kenig, The inhomogeneous Dirichlet problem in Lipschitz domains, J. Funct. Anal. 130, 1 (1995), 161-219.

[24] Alf Jonsson And Hans Wallin, Function spaces on subsets of $\mathbf{R}^{n}$, Math. Rep. 2, 1 (1984), xiv+221.

[25] Doyoon Kim, Trace theorems for Sobolev-Slobodeckij spaces with or without weights, J. Funct. Spaces Appl. 5, 3 (2007), 243-268.

[26] G. KYRIAZIS, Decomposition systems for function spaces, Studia Math. 157, 2 (2003), $133-169$.

[27] P. I. LiZORKIn, Boundary properties of functions from “weight” classes, Soviet Math. Dokl. 1 (1960), 589-593.

[28] Svitlana Mayboroda And Marius Mitrea, Sharp estimates for Green potentials on nonsmooth domains, Math. Res. Lett. 11, 4 (2004), 481-492.

[29] Vladimir MaZ'ya, Marius Mitrea, and TATyana ShaposhniKova, The Dirichlet problem in Lipschitz. domains for higher order elliptic systems with rough coefficients, J. Anal. Math. 110 (2010), 167-239.

[30] Norman G. Meyers, An $L^{p}$-estimate for the gradient of solutions of second order elliptic divergence equations, Ann. Scuola Norm. Sup. Pisa (3), 17 (1963), 189-206.

[31] Petru Mironescu And Emmanuel Russ, Traces of weighted Sobolev spaces. Old and new, Nonlinear Anal. 119 (2015), 354-381.

[32] Irina Mitrea And Marius Mitrea, Boundary value problems and integral operators for the biLaplacian in non-smooth domains, Atti Accad. Naz. Lincei Rend. Lincei Mat. Appl. 24, 3 (2013), 329-383.

[33] IRINA Mitrea AND Marius Mitrea, Multi-layer potentials and boundary problems for higherorder elliptic systems in Lipschitz domains, Lecture Notes in Mathematics, vol. 2063, Springer, Heidelberg, 2013.

[34] Irina Mitrea, Marius Mitrea, And Matthew Wright, Optimal estimates for the inhomogeneous problem for the bi-Laplacian in three-dimensional Lipschitz domains, J. Math. Sci. (N. Y.), 172, 1 (2011), 24-134. Problems in mathematical analysis. No. 51.

[35] S. M. NIKOL'SKIĬ, Priblizhenie funktsii mnogikh peremennykh i teoremy vlozheniya [Approximation of functions of several variables and imbedding theorems ]. "Nauka", Moscow, 1977. Second edition, revised and supplemented.

[36] S. M. Nikol'skiĬ, P. I. Lizorkin, And N. V. Miroshin, Weighted function spaces and their applications to the investigation of boundary value problems for degenerate elliptic equations, Izv. Vyssh. Uchebn. Zaved. Mat. 8 (1988), 4-30. 
[37] JaAk PeEtre, New thoughts on Besov spaces, Mathematics Department, Duke University, Durham, N. C., 1976. Duke University Mathematics Series, no. 1.

[38] S. Rolewicz, On a certain class of linear metric spaces, Bull. Acad. Polon. Sci. Cl. III., 5 (1957), 471-473, XL.

[39] Thomas Runst And Winfried SiCKel, Sobolev spaces of fractional order, Nemytskij operators, and nonlinear partial differential equations, de Gruyter Series in Nonlinear Analysis and Applications, vol. 3, Walter de Gruyter \& Co., Berlin, 1996.

[40] V. V. SHAN' KOV, The averaging operator with variable radius, and the inverse trace theorem, Sibirsk. Mat. Zh. 26, 6 (1985), 141-152, 191.

[41] Elias Stein, Singular integrals and differentiability properties of functions, Princeton Mathematical Series, no. 30, Princeton University Press, Princeton, N. J., 1970.

[42] HAns TRIEBEL, Interpolation theory, function spaces, differential operators, North-Holland Mathematical Library, vol. 18, North-Holland Publishing Co., Amsterdam, 1978.

[43] Hans Triebel, Theory of function spaces, Monographs in Mathematics, vol. 78, Birkhäuser Verlag, Basel, 1983.

[44] S. V. UsPENSKIĬ, Imbedding theorems for classes with weights, Trudy Mat. Inst. Steklov. 60 (1961), 282-303.

[45] Gregory Verchota, The biharmonic Neumann problem in Lipschitz domains, Acta Math. 194, 2 (2005), 217-279.

[46] GREGORY VERCHOTA, Boundary coerciveness and the Neumann problem for 4th order linear partial differential operators, in Around the research of Vladimir Maz'ya. II, Int. Math. Ser. (N. Y.), vol. 12, pages 365-378. Springer, New York, 2010.

[47] Daniel Zanger, The inhomogeneous Neumann problem in Lipschitz domains, Comm. Partial Differential Equations 25, 9-10 (2000), 1771-1808. 